\title{
Research and Development of Antibacterial Metallic Materials
}

\author{
Yuan Junping \\ Jewelry Institute \\ Guangzhou Panyu polytechnic \\ Guangzhou, China \\ yuanjp@gzpyp.edu.cn \\ Chen Lingxia \\ Jewelry Institute \\ Guangzhou Panyu polytechnic \\ Guangzhou, China \\ chenlx@gzpyp.edu.cn
}

\author{
Huang Yuheng, Chen Lingxia \\ Jewelry Institute \\ Guangzhou Panyu polytechnic \\ Guangzhou, China \\ huangyh@gzpyp.edu.cn
}

\begin{abstract}
There are huge demands for antibacterial metallic materials in protecting human health from bacteria propagation and bacteria spreading, and in preventing industrial equipments from microbial corrosion. In this paper, some common inorganic antibacterial agents such as $\mathrm{Ag}^{+}, \mathrm{Cu}^{2+}, \mathrm{Zn}^{2+}$ and $\mathrm{Ce}^{2+}$ were introduced; the manufacturing approaches of the antibacterial metallic materials were analyzed from three types, including the overall alloying, the surface modification and the composite materials. Based on this, the development tendency of the antibacterial metallic materials were discussed, it is advised that the evaluation of one manufacturing approach should be determined from the actual requirements, and the antibacterial theory and evaluation criteria for antimicrobial performance should be further studied.
\end{abstract}

Keywords-Microbiological corrosion; Antibacterial; metallic material; antibacterial agent; manufacturing approaches

\section{BACTERIA PROPAGATION AND MICROBIOLOGICAL CORROSION}

Bacteria propagation and bacteria spreading pose a serious threat to human health, such as the disastrous occurrence of E. Coli and Methicillin-Resistant Staphylococcus Aureus (MRSA) in Japan in 1996, the outbreak of SARS virus infection at a large scale in China in 2003, and the outbreak of the deadly epidemic hemorrhagic E. Coli in German in 2011. All of those serious bacterial infections arouse the consciousness of human hygiene and human health and promote the development and progress of antibacterial materials ${ }^{[1]}$.

Microbiological corrosion is a form of corrosion of metallic materials occurring in the microbial environment, which is the influence of microbial life activities indirectly on the electrochemical process of metal corrosion. The key lies in the interaction between the biological membrane and metallic matrix [2]. Microbiological corrosion causes a different degree of losses to the industrial equipment, civil engineering and aerospace and shipping machine, especially the disastrous losses of the petrochemical and power equipment.

\section{INORGANIC ANTIBACTERIAL AGENTS}

The chemical agent can make the microbial growth or reproduction remain in a certain level within a certain period of time, which is known as the antibacterial agent. The antibacterial agents can be divided into organic antibacterial agent and inorganic antibacterial agent. The organic antibacterial agent can effectively inhibit harmful bacteria and the production and reproduction of the myocyte. It is effective compared to its poor thermal stability, easy decomposition, poor durability, serious toxicity, and hazard to the human body in the long time usage. The inorganic antibacterial material can be further subdivided into the photocatalytic materials, materials containing metal ion and metal oxide. Nowadays, the antibacterial agent of inorganic antibacterial material is mainly metal ion.

With the consideration of the security and the antibacterial property, the best choice is $\mathrm{Ag}^{+}$, followed by $\mathrm{Cu}^{2+}$ and $\mathrm{Zn}^{2+}$. Currently, the most comprehensive used is the type of $\mathrm{Ag}, \mathrm{Cu}$, and $\mathrm{Zn}$. Furthermore, it has been reported that $\mathrm{Ce}$ can be treated as the antibacterial stainless steel in the main antibacterial agents.

\section{THE MANUFACTURING APPROACHES OF THE ANTIBACTERIAL METALLIC MATERIALS}

\subsection{The overall antibacterial metallic materials}

It is an important direction of research on antibacterial metal to prepare antibacterial metallic materials by alloying, which can directly work as matrix materials with excellent antibacterial properties of the metal, such as Ag alloy, $\mathrm{Cu}$ alloy. Moreover, the metal materials without antibacterial properties can add alloying elements to alloying, including $\mathrm{Ag}, \mathrm{Cu}$, zinc and rare earth. And some antibacterial property can be obtained by proper heat treatment. One of the most extensive range of applications and most social effect of the overall antibacterial metallic materials is the antibacterial stainless steel. However, the $\mathrm{Cu}$ alloy is used to replace the stainless steel to reduce the risk of food contamination and nosocomial infection in the hospital equipment. What's more, the cobalt-base 
alloy and the titanium alloy are conducted with the antibacterial modified process. That is to say, they have the property of the anti- microbiological corrosion, which has become the research focus in the field of biomedical materials.

\subsubsection{Copper and its alloy}

Mehtar et al. ${ }^{[3]}$ comparatively research on the resistance capacity of the $\mathrm{Cu}$, stainless steel and PVC to Methicillin S. Aureus, Klebsiella Pneumoniae and Pseudomonas Aeruginosa, which find that the content of $\mathrm{Cu}$ in the brass and Alpaka is more than 55\%, which can effectively inhibit the activity of the pathogenic bacteria in hospitals while the stainless steel and PVC showed no antibacterial properties. USA Environmental Protection Bureau recently approves the $\mathrm{Cu}$ as the first solid antibacterial material. The $\mathrm{Cu}$ used in the door handle, bathroom appliances, and the bed railing helps to control infection in hospitals.

\subsubsection{The overall antibacterial stainless steel}

(1) The Cu-bearing antibacterial stainless steel

The traditional stainless steel has no antibacterial property. When it is added with a certain amount of $\mathrm{Cu}$ with the antimicrobial efficacy, it also does not exhibit antimicrobial property when the $\mathrm{Cu}$ dissolves in the matrix. Furthermore, it is Japan to first discover that the $\mathrm{Cu}$-bearing ferrite stainless steel can obtain excellent antibacterial property with the aging heat treatment for a long time ${ }^{[4]}$.

Some researchers have carried out an in-depth study in the $\mathrm{Cu}$-bearing antibacterial stainless steel [5], which determines that the antibacterial property drives from the $\varepsilon-\mathrm{Cu}$ precipitated phase from the antibacterial heat treatment. The parameters of the antibacterial treatment have obvious influence on the morphology and the amount of the precipitated phase. The Cu-bearing antibacterial stainless steel has performed a good antibacterial function, which has over $99 \%$ sterilizing rate to the E. Coli, S. Aureus, and Candida Albicans.

Generally speaking, the production process of the $\mathrm{Cu}$-bearing antibacterial stainless steel is complicated, which will have adverse effects on the corrosion resistance of the stainless steel. Unfortunately, if the $\mathrm{Cu}$ content is too high, it will impact on the thermal, mechanical properties of the stainless steel. Therefore, the stainless steel with $\mathrm{Cu}$ as the main antibacterial elements needs to obtain optimal matching in the corrosion resistance, the antibacterial properties, and the processing properties.

(2) The Ag-bearing antibacterial stainless steel

Yokota et al. ${ }^{[6]}$ found that, by adding vanadium, limiting impurity of the sulfur content, and getting command of casting speed in the Ag-bearing stainless steel, the distribution status of Ag can be improved in the stainless steel matrix, and an excellent antibacterial performance can be obtained. When the Ag content is lower than $0.001 \%$, there is no obvious antibacterial effect. However, more than $0.3 \%$ of the Ag content will reduce the corrosion resistance of stainless steel and increase the defects in the hot rolling process, which is unfavorable to the cost. The study of Liao et al. ${ }^{[10]}$ finds that the $\mathrm{Ag}$ added 304 stainless steel is composed by $\alpha, \gamma$ and abundant Ag phases, the proportion of $\alpha$ phase will increase with the increase of the Ag content, Ag-bearing stainless steel has excellent antimicrobial properties against E. Coli.

Restricted by the low solubility of $\mathrm{Ag}$ in the iron-base alloy, the development of the Ag -bearing antibacterial stainless steel is generally regarded as very difficult thing. With the improvement of the level of awareness and the means of production, the Ag-bearing antibacterial stainless steel has become a new research focus. Since Ag belongs to the rare and precious metals, it needs to solve the problems in the development of Ag-bearing antibacterial stainless steel, such as how can we use a trace of $\mathrm{Ag}$ alloy to obtain an excellent antibacterial performance and still maintain the original corrosion resistance and processing properties of the stainless steel.

(3) The Ce-bearing antibacterial stainless steel

Rare earth composites have the antibacterial, bactericidal and anti-inflammatory effects. And it has a wide application in the inorganic antibacterial materials ${ }^{[9]}$. In recent years, domestic and foreign researchers utilize rare earth elements to modify the properties of the stainless steel in order to acquire the necessary antibacterial properties. Jing and Min et al ${ }^{[10]}$ study the Ce $0.05-4.5 w t \%$ bearing antibacterial stainless steel. Allegedly, this kind of stainless steel can obtain favorable antibacterial properties without special heat treatment. Moreover, it has the advantages of the long-lasting antibacterial performance, a wide antibacterial range and no-resistance produced by the bacteria.

A trace of the rare earth elements is beneficial to improve the metallurgical quality of the stainless steel. However, when the content exceeds a certain value, rare earth and rare earth metal alloy elements will generate rare earth - intermetallic composite distributed along crystal boundary, which will pose a serious impact on the thermo plasticity of the stainless steel ${ }^{[11]}$. Therefore, the stainless steel with rare earth as the main antibacterial elements needs to take the optimal matching in the antibacterial properties and the processing properties into consideration.

\subsubsection{The overall antibacterial cobalt alloy}

Cobalt based alloy is a kind of important biomedical material. Huang Yongling et al ${ }^{[12]}$ research on the corrosion of Co-Cr-Mo alloy artificial joints. They find green pigment grume produced by Pseudomonas Aeruginosa in the metabolic process attaches to the metal surface. Moreover, they also recognize that produce superoxide ion $\left(\mathrm{O}^{2-}\right)$ with high oxidative capacity is produced in the metabolic process, making the artificial joint of the Co-Cr-Mo porous surface generate the corrosion and lose efficacy.

Faced with the problem of microbial corrosion of cobalt alloy in the long-term implantation into the human body, Toyonaka et al ${ }^{[13]}$ invent the cobalt based alloy material, which can have the antibacterial properties with the dissolution of cobalt ions. The addition of iron, nickel, $\mathrm{Cu}$ and other elements has improved the processing performance, which can be used in the production of the complex shape workpiece. But it needs to point out that cobalt ion itself has limited antibacterial properties and easily leads to allergies. Therefore, the modification of the cobalt alloy is to make it have good antibacterial properties with the precondition of maintaining excellent corrosion resistance, which become an inevitable issue in 
the usage of the material in the microbial environment. Up to now, the antibacterial effect of cobalt alloy can basically achieve by the surface modification. However, there is almost no report related to the overall antibacterial cobalt alloy.

\subsubsection{The overall antibacterial titanium alloy}

The titanium alloy has outstanding biocompatibility and corrosion resistance. If it is used as human implant material, what the anti-adhesion properties of microorganisms and microbial resistant corrosion are will attach great attention from people. Shi et al. ${ }^{[14]}$ state briefly that, the implantation of the titanium alloy into the human body will arouse the situation of bacterial adhesion, which has the risk for infection. With the help of graft modification to titanium alloy surface, the bacterial adhesion is greatly reduced. So far, antibacterial effect obtained by the titanium alloy is basically achieved by the surface modification. What's more, there is a little researches and reports on the overall antibacterial titanium alloy.

\subsection{Surface antibacterial metallic materials}

In the reaction of the biological environment and the metal material, the surface of the material plays a very important role. Therefore, it has become an important way to improve the use effect of the material through surface modification to control structure and properties of the surface material ${ }^{[15]}$. According to the forming mechanism of antibacterial modification layer in the material surface, it can be divided into the mechanical method, the chemical / electrochemical method and the physical method, including physical vapor deposition, ion implantation and solid infiltration. As for the solid infiltration, it includes sol-gel method, chemical vapor deposition and electrochemical deposition and so on ${ }^{[16]}$.

\subsubsection{Physical vapor deposition}

Physical vapor deposition is to put the target materials for evaporation or sputtering in vacuum environment. The migration is in the forms of atoms, molecules or ions state to deposit and form a film on the matrix surface. Zhang Xiangyu et al ${ }^{[17]}$ apply the alloying of the plasma surface and the composite processing technology of the glow bombardment for the heat diffusion into the $\mathrm{Cu}$ alloy processing in the stainless steel surface, which obtains the $\mathrm{Cu}$ alloy layer with the amount of $5.7 \mathrm{wt} \% \mathrm{Cu}$ and thickness of about $2.7 \mu \mathrm{m}$. After contacting with the bacteria liquid, the $\mathrm{Cu}$ element of the stainless steel surface precipitates as the $\mathrm{Cu}$ ion, which shows excellent antibacterial properties to the E. Coli and S. Aureus.

\subsubsection{Ion implantation}

Ion implantation is the method of accelerating the high-energy ion implantation into the solid surface under vacuum condition. The ion implantation technology is to implant the $\mathrm{Ag}$ ion and $\mathrm{Cu}$ ion on the surface of the metal base, causing the material with the necessary antibacterial performance, which has become a focus of researches. Shinonaga et al. ${ }^{[18]}$ utilizes the plasma source to implant fluorine and $\mathrm{Ag}$ ions in the organic glass surface, which has obtained good antibacterial effect. Ni et al [19] implants $\mathrm{Ag}$ ion and $\mathrm{Cu}$ ion on the 420 stainless steel surface, when the two icons reach the total dose of $2 \times 10^{17}$ ions $/ \mathrm{cm}^{2}$, the materials have excellent performance of antibacterial properties to the E. Coli and S. Aureus.

\subsubsection{Solid infiltration method}

Li Dong et al ${ }^{[20]}$ adopt deposition diffusion method to produce Antibacterial $\mathrm{Cu}$ layer with $\varepsilon-\mathrm{Cu}$ phase in 0Cr18Ni9 austenitic stainless steel and $2 \mathrm{Cr} 13$ martensite stainless steel surface, which possess good antimicrobial properties against E. Coli and S. Aureus.

\subsubsection{Sol-gel method}

It takes the metal alkoxide as the precursor, the substrate material gets into the solution containing the precursor and pull through with a certain speed. With solvent volatilization, the solution gets roasting and drying and forms a layer of thin film on the surface of the substrate. By this means to prepare the $\mathrm{TiO}_{2}$ and $\mathrm{SiO}_{2}$ thin films to obtain good antibacterial properties ${ }^{[21]}$. Jeon et al ${ }^{[22]}$ prepare the $\mathrm{Ag}$ doped $\mathrm{SiO}_{2}$ film by sol-gel method. After annealing of $600^{\circ} \mathrm{C}, \mathrm{Ag}$ is completely entrapped in the $\mathrm{SiO}_{2}$ matrix, and antibacterial ability to E. Coli and $\mathrm{S}$. Aureus in the film almost reaches $100 \%$.

\subsubsection{Chemical vapor deposition method}

The chemical vapor deposition method, developed in recent decades, is a new technique of preparing the inorganic material. In recent years, it has also been widely used for the preparation of antibacterial films ${ }^{[23]}$. Marciano et al. ${ }^{[24]}$ use the PECVD process for the deposition DLC films doped with Ag on the surface of 316L stainless steel, while the film structure is not changed due to the mingle of the Ag. However, the compression stress of the film decreases significantly with the increasing amount of $\mathrm{Ag}$ nanoparticles layer. DLC film doped with Ag exhibits good antibacterial properties on the E. Coli, which can be applied in biomedical field.

\subsection{Composite antibacterial metallic materials}

Japan has developed a composite antibacterial stainless steel plate with excellent antibacterial properties. It is composed of 5 layer plates, the core material is the AUS8 stainless steel with high carbon martensitic system tool to ensure the sharp blade, and the surface plate uses the SUS410 stainless steel with good corrosion resistance of low carbon martensitic system. The $\mathrm{Cu}$ or $\mathrm{Cu}$ alloy plate having antibacterial property is installed between the core plate and the surface plate. What's more, the five layers of the plates are rolled into the Cutting tool with antibacterial composite stainless steel plate ${ }^{[25]}$

\section{THE DEVELOPMENT TENDENCY OF THE ANTIBACTERIAL METALLIC MATERIALS}

As for the distribution of the antimicrobial agent, antibacterial metallic materials can be divided into the overall type, surface type and composite type, taking the former two kinds as the main types. In terms of ways of producing antibacterial effect, it can be divided into ion dissolution type and photocatalysis type. The overall antibacterial metallic materials belong to the type of the ion dissolution, which occupy the majority share of antibacterial metallic materials. There is the distribution of antibacterial metallic element inside and surface of the overall antibacterial metallic materials, which will not lost the antibacterial performance for the surface wear with the virtues of durable antibacterial properties and stability. Through the interaction of the dissolution of metal ions from the material surface and bacteria, it inhibits the metabolism and reproduction of bacteria. Therefore, the dissolution of metal ions is the premise to obtain the 
antibacterial effect. However, it will have risk of partly damaging or deteriorating surface passivation film of the metal material to decrease the corrosion resistance. Consequently, in the research and development of the overall antibacterial metallic materials, it is very important to choose appropriate antimicrobial elements with the priority of the strong antibiosis as well as high security elements to make the uniform distribution in the material matrix structure. Surface antibacterial metallic materials have the dissolution of metal ions as well as the photocatalysis type of which the former has mechanism of antibacterial action with the overall type; the latter is mostly represented by the anatase $\mathrm{TiO}_{2}$ thin film. Under the exposure of sun or illumination, it forms reactive oxygen substances composed by the oxygen in the air or moisture to play the bactericidal role. In this way, its antibacterial effect belongs to the immediate action. Compared with the antibacterial agents consuming the Ag, $\mathrm{Cu}$ and other metal ions, it has the advantages. Nonetheless, if there is no light, it will fail to produce antibacterial efficacy. In order to overcome this disadvantage, the $\mathrm{TiO}_{2}$ thin film is mingled with $\mathrm{Ag}, \mathrm{Cu}$ and other metal ions, which will make it with antibacterial efficacy of photocatalysis and the dissolution of metal ions. Therefore, it has become the research focus of the surface antibacterial metallic materials. Compared with the overall antibacterial metallic materials, the surface antibacterial metallic material has the advantages of quick effect, less consumption, and wide application in the substrates of all kinds of materials. Due to the thin surface film layer results in the antimicrobial effect, the antibacterial properties will lost once the thin surface film layer wears. Therefore, in the research and development of antibacterial metallic materials, we should not simply think the overall type is better than the surface type, or vice versa, and we should determine it based on the actual requirements.

Since the inorganic antibacterial agent has been developed for a short time, the antibacterial mechanism is still in the stage of exploration and does not have uniform standard on the evaluation of the antibacterial performance. Moreover, the research of antibacterial materials involves multiple disciplines, including biology and material science. It needs a long time before establishing the knowledge structure of the comprehensive system, which will influence on the study process to some extent. Especially, it still needs further study on the antibacterial theory and evaluation criteria for antimicrobial performance.

\section{REFERENCES}

[1] Li Mei, Wang Qing-rui. The development and application of antibacterial materials [J]. New Chemical Materials, 1998,5: 8-11(in Chinese).

[2] Hector A. Videla, William G. Characklis. Biofouling and microbially influenced corrosion $[\mathrm{J}]$. International Biodeterioration \& Biodegradation, 1992,29(3-4): 195-212.

[4] S. Mehtar, I. Wiid, S.D. Todorov. The antimicrobial activity of copper and copper alloys against nosocomial pathogens and Mycobacterium tuberculosis isolated from healthcare facilities in the Western Cape: an in-vitro study[J]. Journal of Hospital Infection, 2008,68(1): 45-51.

[5] S Nakamura, S Suzuki, N Ookubo. Microstructure and antimicrobial activity of $\mathrm{Cu}$ contained ferritic stainless steels[J]. Camp-ISIJ, 1998,11(3): 1147 (in Japanese)

[6] Ling Ren, Ke Yang, Lei Guo, et al. Preliminary study of anti-infective function of a copper-bearing stainless steel [J]. Materials Science and Engineering: C, 2012,32(5): 1204-1209.

[7] Takeshi Yokota, Misako Tochibara, Masayuki Ohta. Ag dispersed stainless steel with antibacterial property[R]. Kawasaki steel technical report, 2002,46: 37-41.

[8] $\mathrm{KH}$ Liao, $\mathrm{KL} \mathrm{Ou}, \mathrm{HC}$ Cheng, et al. Effect of $\mathrm{Ag}$ on antibacterial properties of stainless steel[J]. Applied surface science, 2010,256(11): 3642-3646.

[9] Chen Ai-mei, Shi Qing-shan, Ouyang You-sheng, et al. Advance research on antibacterial effect and application of rare earth $[\mathrm{J}]$. Microbiology, 2009, 36(1): 90-96. (in Chinese)

[10] Jing He-min, Wu Xin-qiang, Liu, Yong-qiang, et al. Study on antimicrobial properties of Ce-bearing antibacterial stainless steel. Journal of Rare Earths[J]. 2006, 24(2): 223-226. (in Chinese)

[11] Tong Shi-hua, Gao Guo-zhong, He Wei-yong, et al. Effects of Ce, yttrium, mischmetal on the thermo plasticity of stainless steel 316L[J]. Chinese Rare Earths, 1983,2: 8-13. (in Chinese)

[12] Huang Yong-ling, Liu Xiao-fang. Microbiological corrosion of CoCr- Mo Alloys [J]. Journal of Materials Engineering, 1997,8: 47-49. (in Chinese)

[13] Isamu Kato Toyonaka, Sadayuki Yubda Suita, Naoki Oda Nishinomiya, et al. Antibiotic alloys [P]. USA patent 4822567, 1989-04-18.

[14] Zhilong Shi, K. G. Neoh, E. T. Kang, et al. Bacterial adhesion and osteoblast function on titanium with surface-grafted chitosan and immobilized RGD peptide[J]. Journal of Biomedical Materials Research Part A, 2008,86A(4):865-872.

[15] Krasimir Vasilev, Jessica Cook, Hans J Griesser. Antibacterial surfaces for biomedical devices[J]. Expert Review of Medical Devices, 2009,6(5): 553-567.

[16] Wang Zhang-ming, Sun Wei, Tian Xiu-bo, et al. Progress in antibacterial field by surface modification [J]. Journal of Materials Science and Engineering, 2006, 24(5): 787-791. (in Chinese)

[17] Zhang Xiang-yu, Jiang Li, Huang Xiao-bo, et al. Improvement of antibacterial properties of stainless steel by combining plasma $\mathrm{Cu}$ and thermal diffusion [J]. Journal of Inorganic Materials, 2012, 27(5): 519-523. (in Chinese)

[18] Yukari Shinonaga, Kenji Arita. Antibacterial effect of acrylic dental devices after surface modification by fluorine and $\mathrm{Ag}$ dual-ion implantation [J]. Acta Biomaterialia, 2012, 8(3):1388-1393.

[19] Hong-wei Ni, Han-shuang Zhang, Rong-sheng Chen, et al. Antibacterial properties and corrosion resistance of AISI 420 stainless steels implanted by $\mathrm{Ag}$ and copper ions[J]. International Journal of Minerals, Metallurgy, and Materials, 2012,19(4): 322-327.

[20] Li Dong, Xu Bo-fan, Ni Hong-wei, et al. Antibacterial copperized layer on stainless steel surface prepared by deposition-diffusion method[J]. Heat Treatment of Metals, 2005, 30(2): 8-11. (in Chinese)

[21] Yang Hui, Miao Ya-mei, Ding Xin-geng. Research and prospect of preparation of functional thin films by sol- gel method [J]. Rare Metal Materials and Engineering, 2004, 33(suppl3): 208-213.

[22] Hyung-Jun Jeon, Sung-Chul Yi, Seong-Geun Oh. Preparation and antibacterial effects of $\mathrm{Ag}-\mathrm{SiO}_{2}$ thin films by sol-gel method [J] Biomaterials , 2003 , (24): 4921-4928.

[23] Susan Krumdieck. Biomaterials by CVD Methods, Introduction to the CVD Special Section[J]. Chemical vapor deposition, 2010,16(1-3): 9-11.

[24] F.R. Marciano, L.F. Bonetti, L.V. Santos, et al. Antibacterial activity of DLC and Ag-DLC films produced by PECVD technique Diamond and Related Materials[J]. 2009,18(5-8): 1010-1014.

[25] By Hiroshi in. Tool for antibacterial stainless steel cladding [J]. Special steel, 1999,48(6): 20-21. (in Japanese). 